\title{
Effets de la perfusion intensive sur quelques paramètres sanguins chez des veaux atteints de diarrhée aiguë
}

Mahangaiko M

Université de Lubumbashi, Lubumbashi Katanga B.P. 1825, République Démocratique du Congo

Auteur correspondant : mahangaikofr@yahoo.fr

Original submitted in on 9th June 2016. Published online at www.m.elewa.org on 31st August 2016 http://dx.doi.org/10.4314/jab.v104i1.6

\section{RESUME}

Objectif : l'objectif de cette étude est d'évaluer les effets d'une perfusion intensive chez des veaux atteints de diarrhée aiguë sur les paramètres sanguins notamment l'hématocrite, les protéines totales, la créatinine et l'urée plasmatiques, le pH, l'excès de base (BE) et le bicarbonate dans le sang.

Méthodologie et résultats : quarante neuf veaux atteints de diarrhée aiguë ont été traités intensivement avec 10 litres de solution de réhydratation pendant 24 heures. Parmi ces veaux, 41 ont été guéris, 5 sont morts de diarrhée et 3 d'autres causes. L'urée, la créatinine, les protéines totales, le pH, l'hématocrite, l'excès de base (BE) et le bicarbonate ont été mesurés avant le traitement, 24 heures après la perfusion et 2 jours après la fin de la diarrhée. L'hématocrite, le pH, l'excès de base et le bicarbonate sont redevenus normaux 24 heures après la perfusion.

Conclusion et application : cette étude a montré qu'une perfusion intensive chez des veaux atteints de diarrhée aiguë permet une réhydratation rapide et retourne en 24 heures l'hématocrite, le $\mathrm{pH}$, l'excès de base et le bicarbonate aux valeurs normales et que les protéines totales, l'urée et la créatinine nécessitent un temps plus long pour se normaliser. Ainsi donc, les praticiens peuvent facilement composer cette solution à administrer aux veaux atteints de diarrhée.

Mots clés : diarrhée, réhydratation, paramètres sanguins

\section{Effects of intensive infusion on some blood parameters in calves with acute diarrhea ABSTRACT}

Objective : the objective of this study is to evaluate the effects of intensive infusion in calves with acute diarrhea on blood parameters including hematocrit, total protein, plasma creatinine and urea, $\mathrm{pH}$, base excess (BE) and bicarbonate in the blood.

Methodology and results: Forty- nine calves with acute diarrhea were treated intensively with $10 \mathrm{I}$ of rehydration solution for 24 hours. Forty-one calves recovered, five died of the diarrhea and 3 from other causes. The urea, creatinine, total protein, $\mathrm{pH}$, hematocrit, base excess $(\mathrm{BE})$ and bicarbonate were measured in the blood before treatment, 24 hours after infusion, and 2 days after the end of the diarrhea. The hematocrit, pH, base excess and bicarbonate returned to normal 24 hours after infusion while urea and creatinine have required time to return to normal. Total protein decreased after infusion to further increase after.

Conclusion and application: This study showed that intensive infusion in calves with acute diarrhea enables rapid rehydration and returns in 24 hours hematocrit, $\mathrm{pH}$, base excess and bicarbonate to normal values and 


\section{Mahangaiko M. J. Appl. Biosci. 2016 Effets de la perfusion intensive sur quelques paramètres sanguins}

chez des veaux atteints de diarrhée aiguë

the total protein, urea and creatinine require a longer time to normalize. The practitioners can easly formulate the solution to be given to the calves with acute diarrhea.

Keys words : diarrhea, rehydration, blood parameters

\section{INTRODUCTION}

La diarrhée chez les jeunes veaux et en particulier celle qui survient durant les deux à trois premières semaines de la vie du nouveau-né constitue l'affection la plus importante par sa fréquence (Trefz et al. 2012). Plusieurs causes sont à l'origine de la diarrhée, qui se caractérise par des matières fécales de consistance liquide. II s'agit d'agents pathogènes (bactéries, virus, protozoaires), des conditions d'élevage, c'est-à-dire un manque d'hygiène qui favorise les agents pathogènes et d'une alimentation inappropriée (Foster et Smith, 2009 ; Al-Robaiee et Al- Farwachi, 2013). Quelque soit la cause de la diarrhée, celle-ci entraine toujours une déshydratation si un apport adéquat en liquide n'est pas maintenu et souvent aussi des troubles biochimiques qui sont considérés comme des causes majeures de la mort de l'animal (Araujo et al. 2015). Les troubles majeurs qui sont habituellement identifiés en cas de diarrhée sont les suivants: déshydratation, acidose, déséquilibre en électrolytes notamment une perte de bicarbonate et une augmentation de potassium et un déficit énergétique une balance énergétique négative ( Trefz et al., 2012, Bednarski et al., 2015). Il arrive aussi dans un

\section{MATÉRIELS ET MÉTHODES}

L'étude a porté sur 49 veaux mâles et femelles de race Pie noire. Ces veaux étaient âgés de 1 à 15 jours et atteints d'une diarrhée aiguë. Seuls les veaux diarrhéiques avec un taux d'urée dans le sang d'au moins $15 \mathrm{mmol} / \mathrm{l}$ ont été considérés dans cette étude. Leur poids était en moyenne de $40,7 \mathrm{~kg}$. Le sang a été prélevé à la veine jugulaire pour différentes analyses. (Coles, 1979). L'hématocrite a été déterminé selon la méthode de micro hématocrite. Les protéines totales, l'urée et la créatinine ont été dosées dans le sérum sanguin par un analyseur automatique Hitachi 705. (Coles, 1979). Le pH sanguin, le taux de bicarbonate dans le sang et l'excès de base (BE) ont été déterminés par un analyseur des gaz (type 855 de la firme CIBA-CORNING). Après ces analyses, les veaux diarrhéiques retenus, c'est-à- dire avec au moins $15 \mathrm{mmol} / /$ d'urée dans le sang ont reçu état avancé de la diarrhée, un dysfonctionnement rénal qui est une cause substantielle de la mort des veaux diarrhéiques (Mechor et al. 1993). Dans une diarrhée, on observe donc une augmentation de taux d'hématocrite, du taux des protéines totales, de l'urée et de la créatinine suite à la diminution de la volémie et l'augmentation de la concentration sanguine (Smith, 2009) et une diminution du pH et du bicarbonate suite à l'acidose (Bednarski et al., 2015). Dans le traitement de la diarrhée, la réhydratation par voie orale ou parentérale reste la mesure la plus importante d'une part pour compenser les pertes et d'autre part restaurer la fonction rénale (Smith, 2009 ; Smith et Berchtold, 2014 ; Trefz et al., 2015). Le but de cette étude est d'évaluer les effets d'une perfusion intensive chez des veaux atteints de diarrhée aiguë sur les paramètres sanguins et notamment l'hématocrite, les protéines totales, la créatinine et l'urée plasmatiques, le $\mathrm{pH}$, l'excès de base $(\mathrm{BE})$ et le bicarbonate dans le sang. Cette perfsusion intensive est une perfusion conçue de manière à donner des résultats plus sensibles.

une solution de perfusion de 101 pendant 24 heures. Cette solution contenait du $\mathrm{NaCl} 0,9 \%$, bicarbonate de sodium $1,3 \%$ et glucose $5 \%$. A la fin de cette perfusion, le sang a été de nouveau prélevé pour déterminer l'hématocrite, les protéines totales, l'urée et la créatinine, le pH, l'excès de base et le bicarbonate dans le sang.Les veaux dont la diarrhée ne s'était pas arrêtée après cette perfusion ont continué à prendre le sérum oral de réhydratation. Deux jours après la fin de la diarrhée qui a été constatée par un retour à la normale de la consistance des matières fécales, le sang a été de nouveau prélevé pour la $3^{\mathrm{ème}}$ fois pour la détermination des mêmes paramètres à savoir l'hématocrite, les protéines totales, l'urée et la créatinine, le pH, l'excès de base et le bicarbonate dans le sang. Le test t de Student a été utilisé pour comparer les moyennes deux à deux. 


\section{RÉSULTATS}

Guérison des veaux : Sur les 49 veaux diarrhéiques qui ont été traités intensivement, 41 ont été guéris, 5 sont morts de diarrhée et 3 d'autres causes. Le rapport entre le délai de guérison et le taux d'urée et de créatinine dans le sang avant le traitement est présenté dans le tableau 1. Le taux d'urée dans le sang n'a pas influencé le délai de guérison car les différences ne sont pas significatives entre les différentes moyennes. II en est de même du taux de créatinine qui n'a pas non plus influencé le délai de guérison même si une tendance à la hausse a semblé être observée chez des veaux qui ont nécessité un délai plus long de guérison. En rapport avec le taux d'hématocrite (Tableau 2), le délai de guérison n'a pas varié avec ce paramètre mesuré avant le traitement.

Tableau 1 : Rapport entre le délai de guérison et le taux d'urée et de créatinine dans le sang avant le traitement

\begin{tabular}{|l|l|l|l|}
\hline Effectif & $\begin{array}{l}\text { Délai de guérison } \\
\text { (jours) }\end{array}$ & $\begin{array}{l}\text { Urée (mmol/l) } \\
(\mathbf{X} \pm \mathbf{S D})\end{array}$ & $\begin{array}{l}\text { Créatinine }(\boldsymbol{\mu m o l} / \mathbf{l}) \\
(\mathbf{X} \pm \mathbf{S D})\end{array}$ \\
\hline 8 & $0-3$ & $25,3 \pm 12,6^{\mathrm{a}}$ & $249,0 \pm 105,8^{\mathrm{b}}$ \\
\hline 19 & $4-10$ & $25,4 \pm 8,5^{\mathrm{a}}$ & $375,6 \pm 179,0^{\mathrm{b}}$ \\
\hline 14 & $>10$ & $29,2 \pm 11,8^{\mathrm{a}}$ & $383,5 \pm 209,8^{\mathrm{b}}$ \\
\hline
\end{tabular}

Les nombres portant en exposant les mêmes lettres dans la même colonne ne sont pas significativement différents au seuil de $5 \%$. Le Tableau 3 donne les valeurs de l'urée et de la créatinine sanguine, de l'excès de base et de l'hématocrite de 5 veaux morts de la diarrhée malgré le traitement.

Tableau 2 : Rapport entre le délai de guérison et le taux d'hématocrite avant le traitement

\begin{tabular}{|l|l|l|}
\hline Effectif & Délai de guérison (jours) & Hématocrite (\%) X $\mathbf{S D}$ \\
\hline 8 & $0-3$ & $39 \pm 9^{*}$ \\
\hline 19 & $4-10$ & $44 \pm 8$ \\
\hline 14 & $>10$ & $45 \pm 9$ \\
\hline
\end{tabular}

${ }^{*}$ : aucune différence significative entre les différentes moyennes au seuil de $5 \%$.

Mortalité des veaux

Tableau 3 : valeurs de l'urée et de la créatinine sanguine, de l'excès de base et de l'hématocrite de 5 veaux morts de la diarrhée

\begin{tabular}{|l|l|l|l|l|}
\hline $\mathbf{N}^{\circ}$ du veau & Urée $(\mathbf{m m o l} / \mathbf{l})$ & Créatinine $(\boldsymbol{\mu m o l} / \mathbf{l})$ & $\mathbf{B E}(\mathbf{m m o l} / \mathbf{l})$ & Hématocrite(\%) \\
\hline 1 & 59,0 & 650,8 & $-21,9$ & 55 \\
\hline 2 & 19,3 & 196,6 & $-7,0$ & 32 \\
\hline 3 & 25,3 & 417,6 & $-6,3$ & 47 \\
\hline 4 & 22,8 & 147,6 & $-15,8$ & 51 \\
\hline 5 & 71,9 & 823,7 & $-13,6$ & 34 \\
\hline$X$ & 39,7 & 447,3 & $-12,9$ & 44 \\
\hline
\end{tabular}

II ressort ici que le veau $\mathrm{n}^{\circ} 5$ a eu un taux d'urée et de créatinine les plus élevés pendant que son taux d'hématocrite ne l'était pas.*

Effet de la perfusion intensive sur certains paramètres sanguins : Le traitement a permis dans un délai de 24 heures de retourner l'hématocrite, le $\mathrm{pH}$, l'excès de base $(\mathrm{BE})$ et le bicarbonate aux valeurs normales. Les protéines totales ont diminué pour augmenter encore un peu tandis que l'urée et la créatinine ont continué à diminuer jusqu'à la guérison (Tableau 4). 


\section{Mahangaiko M. J. Appl. Biosci. 2016 Effets de la perfusion intensive sur quelques paramètres sanguins}

chez des veaux atteints de diarrhée aiguë

Tableau 4 : L'effet de la perfusion intensive sur l'hématocrite, les protéines totales, l'urée et la créatinine, le pH, l'excès de base et le bicarbonate dans le sang pour 30 veaux

\begin{tabular}{|l|c|c|c|}
\hline Paramètres & $\begin{array}{c}\text { Avant traitement } \\
(\mathbf{X} \pm \mathbf{S D})\end{array}$ & $\begin{array}{c}\text { 24 heures après } \\
\text { traitement }(\mathbf{X} \pm \mathbf{S D})\end{array}$ & $\begin{array}{c}\text { Après la guérison } \\
(\mathbf{X} \pm \mathbf{S D})\end{array}$ \\
\hline Hématocrite $(\%)$ & $45 \pm 8^{\mathrm{a}}$ & $33 \pm 6^{\mathrm{b}}$ & $33 \pm 6^{\mathrm{b}}$ \\
\hline Protéines totales $(\mathrm{g} / \mathrm{l})$ & $54,9 \pm 7,9^{\mathrm{a}}$ & $39,8 \pm 4,3^{\mathrm{b}}$ & $47,0 \pm 8,0^{\mathrm{c}}$ \\
\hline Urée $(\mathrm{mmol} / \mathrm{l})$ & $24,2 \pm 8,0^{\mathrm{a}}$ & $9,4 \pm 5,6^{\mathrm{b}}$ & $3,5 \pm 2,0^{\mathrm{c}}$ \\
\hline Créatinine $(\mu \mathrm{mo} / / \mathrm{l})$ & $325,5 \pm 178,0^{\mathrm{a}}$ & $185,6 \pm 174,3^{\mathrm{b}}$ & $86,1 \pm 16,8^{\mathrm{c}}$ \\
\hline $\mathrm{pH}$ & $7,126 \pm 0,160^{\mathrm{a}}$ & $7,340 \pm 0,064^{\mathrm{b}}$ & $7,344 \pm 0,046^{\mathrm{b}}$ \\
\hline $\mathrm{BE}(\mathrm{mmol} / \mathrm{l})$ & $-12,3 \pm 9,0^{\mathrm{a}}$ & $1,8 \pm 6,2^{\mathrm{b}}$ & $2,4 \pm 4,6^{\mathrm{b}}$ \\
\hline Bicarbonate $(\mathrm{mmol} / \mathrm{l})$ & $16,2 \pm 6,6^{\mathrm{a}}$ & $27,2 \pm 5,7^{\mathrm{b}}$ & $28,1 \pm 4,6^{\mathrm{b}}$ \\
\hline
\end{tabular}

Les nombres portant en exposant les mêmes lettres dans la même ligne ne sont pas significativement différents au seuil de $5 \%$.

\section{DISCUSSION}

Un délai de guérison de 7,4 \pm 4 jours a été noté à l'issue de cette étude. Ce délai correspond à celui observé par Niemeyer (1992) qui était de 8,9 jours et par Seeman (1993) qui était de 7,0 + 3,9 jours. Par contre, ce délai de guérison a été supérieur à celui observé par Hartmann et Reder (1995) chez des veaux qu'ils avaient classés de légèrement diarrhéiques et qui était de 3 jours. Au sein des veaux diarrhéiques, il est ressorti que $19,5 \%$ des veaux ont été guéris endéans 3 jours sans que leurs taux d'urée ou d'hématocrite aient été différents de ceux guéris entre 4 et 10 jours ou ceux guéris après 10 jours. Ainsi donc, le délai de guérison n'a pas été lié à la séverité de la maladie. Cette observation correspond aux résultats de Hinderer (1997). La comparaison des différentes moyennes a montré que le délai de guérison n'était lié ni au taux d'urée ni à celui de l'hématocrite et donc pas à la sévérité de la maladie. Mortalité des veaux. Cinq veaux sont morts malgré une perfusion intensive. On pourrait penser que les veaux qui meurent de diarrhée sont ceux là avec un taux d'urée, de créatinine et hématocrite très élevé. Cette étude a montré que paradoxalement, des veaux avec des taux faibles sont morts malgré le traitement et que d'autres avec de taux très élevés ont été guéris. Probablement que d'autres causes non connues pourraient y jouer un rôle. La déshydratation provoquée par la diarrhée entraine une diminution de la fonction rénale et donc un dysfonctionnement de celui-ci. Et il est admis que le

\section{CONCLUSION ET APPLICATION DES RESULSTATS}

Cette étude a montré qu'une perfusion intensive chez des veaux atteints de diarrhée aigüe, permet une réhydratation rapide et retourne en 24 heures l'hématocrite, $\mathrm{pH}$, excès de base et bicarbonate aux valeurs normales et que les protéines totales, l'urée et la dysfonctionnement rénal est une cause majeure de la mort des veaux diarrhéiques (Kholer et al., 2015). Une correction de cette fonction devrait soulager les animaux malades et apporter leur guérison. II apparait donc que d'autres causes non connues sont responsables de la mort des veaux diarrhéiques. Concernant l'effet de la perfusion intensive sur les paramètres sanguins étudiés : Le taux d'hématocrite, le pH, l'excès de base et le taux de bicarbonate sont redevenus normaux 24 heures après la perfusion. Les valeurs obtenues après la perfusion n'étant pas significativement différentes de celles observées après la guérison. Le retour à la normale de l'hématocrite après cette perfusion suggère que cette réhydratation a pu corriger la déshydratation, le taux d'hématocrite étant un indice du degré de déshydratation (Khan et Zaman, 2010). Par contre, le taux de l'urée et de la créatinine dans le sang n'ont pas atteints leurs valeurs normales après cette perfusion. Ces taux bien que sensiblement diminués après la perfusion sont restée élevés. L'urée et la créatinine étant des substances éliminées par les reins, il apparait ici que dans une azotémie pré-rénale comme c'est le cas, la fonction rénale nécessite un certain temps pour être pleinement fonctionnelle malgré une volémie normale. Les protéines totales ont diminué pour augmenter légèrement par la suite. Leur diminution après la perfusion peut être la conséquence de l'augmentation de la volémie (Berchtold, 2009).

créatinine nécessitent un temps plus long pour se normaliser. Ainsi donc, les praticiens peuvent facilement composer cette solution à administrer en cas de diarrhée aiguë chez les veaux. 


\section{REFERENCES}

Al-Robaiee IA and Al-Farwachi MI, 2013. Prevalence of rotaviral infection in diarrheic neonatal calves in Mosul city, Iraq. Veterinary World, 6:538-540

Araujo G, Yunta C, Terré M, Mereu A, Ipharraguerre I, Bach A, 2015. Intestinal permeability and incidence of diarrhea in newborn calves. J Dairy Sci., 15: 514-517

Bednarski M, Kupczynski R, Sobiech P, 2015. Acid-base disorders in calves with chronic diarrhea. Pol J vet Sci., 18: 207-215

Berchtold J, 2009. Treatment of calf diarrhea: intravenous fluid therapy. Vet Clin North Am Food Anim Pract 25 : 73-99

Coles EH, 1979. Le laboratoire en clinque vétérinaire. Editions Vigot, Paris

Foster DM and Smith GW, 2009. Pathophysiology of diarrhea in calves. Vet Clin North Am Food Anim Pract, 25: 13-36

Hartmann $H$ et Reder S, 1995. Einfluss von Dehydratation auf funktionlle Parameter des Flüssigkeitshaushaltes sowie Wirksamkeit einer Rehydratation mit kristalliner oder kolloidaler Infusionslösung bei Kälbern. Tierärztl. Prax. 23: 342-350

Hinderer A, 1997. Vergleich von Ad Libitum-und rationierter Milchtränkung bei jungen Kälbern mit akutem durchfall. Vet. Med. Diss. München

Khan A and Zaman T, 2007. Effects of rehydration solution on hematological and biochemical parameters in induced buffalo neonatal calf diarrhea. Ital.J.Anim.Sci., 6 : 957-960

Kohler GD, Gaut JP, Morrison AR, 2015. The Case Diarrhea, weight loss, electrolyte abnormalities, and renal failure. Kidney Int., 2: 421-432

Mechor GD, Cebra C, Blue J, 1993. Renal failure in a calf secondary to chronic enteritis. Cornell Vet., 83: 325-331

Niemeyer C, 1992. Untersuchungen zur Diätregelung bei neonatalen Diarrhoe des Kalbes : Beziehungen zwichen Tränkeverfarhren und Krankheitverlauf. Vet. med. Diss. München.

Seeman G, 1993. Untersuchung über die therarpeutische Wirksamkeit des Huminsäuren-Präparates "Dysticum" bei der Neugeborenendiarrhoe des Kalbes. Vet.med. Diss. München

Smith GW, 2009. Treatment of calf diarrhea : oral fluid: Vet Clin North Am Food Anim Pract, 25 : 55-72

Smith GW and Berchtold J, 2014. Fluid therapy in calves. Vet Clin North Am Food Anim Pract 30 :409-427
Trefz FM, Lorch A, Feist M, Sauter-Louis C, Lorenz I, 2012. Construction and validation of a decision tree for treating metabolic acidosis in calves with neonatal diarrhea. BMC Vet Res.,8: 238- 245

Trefz FM, Lorch A, Zitzl J, Kutschke A, KnubbenSchweizer G, Lorenz I, 2015. Effects of alkalinization and rehydration on plasma potassium concentrations in neonatal calves with diarrhea. J Vet Intern Med., 2: 696-704 